\title{
Dissonance Engineering: A New Challenge to Analyse Risky Knowledge When using a System
}

\author{
F. Vanderhaegen
}

\author{
Frédéric Vanderhaegen \\ ${ }^{1}$ Univ Lille Nord de France, F-59000 Lille, France \\ ${ }^{2}$ UVHC, LAMIH, F-59313 Valenciennes, France \\ ${ }^{3}$ CNRS, UMR 8201, F-59313 Valenciennes, France \\ frederic.vanderhaegen@univ-valenciennes.fr
}

\begin{abstract}
The use of information systems such as on-board automated systems for cars presents sometimes operational risks that were not taken into account with classical risk analysis methods. This paper proposes a new challenge to assess risks by implementing an automated tool based on the dissonance engineering principle. It consists in analysing knowledge in term of dissonances. A dissonance is defined as a knowledge that sounds wrong, or in other words that may present conflicts. The paper focuses on two kinds of dissonances: erroneous affordances when events can be related to erroneous actions and contradictory knowledge when the application of knowledge relates to opposite actions. The proposed automated tool analyses the knowledge base content in order to detect possible dangerous affordances or contradictory knowledge. An example of application is given by using a limited number of simple rules related to the use of an Automated Speed Control (ASC) system for car driving.

Keywords: dissonance engineering, erroneous affordance, contradictory knowledge, risk analysis, car driving system.
\end{abstract}

\section{Introduction}

Is there something wrong when engineers or researchers design walking robots directly with two legs without copying the learning process of the human walking that begins initially with the legs and the hands, then with the use of supports and finally with both legs after the complete control of the equilibrium? To control such a process, undesirable events such as lack of knowledge to control equilibrium or breakdown of equilibrium or fatal fall should be studied in order to design algorithms or other devices that are able to prevent the walking robots from a loss of their equilibrium.

Classical risk analysis focuses on the identification and the control of such undesirable events and aims at providing the human-machine systems with barriers in order to protect them from the occurrence or the impact of these events [1]. Despite these barriers, accidents remain and retrospective analyses can help the designers to identify what was wrong. Safety based analysis can apply different methods. The RAMS based methods (Reliability, Availability, Maintainability and Safety based analyses) treat about technical failures. The methods from cindynics treat about organizational dangers Human reliability or human error based analyses focus on the success or the failure of human behaviours respectively. Resilience or vulnerability based methods consider the analysis of the success or the failure of the control of the system stability respectively. This paper proposes on a new way to analyse risks: the use of the dissonance concept to assess conflicts between knowledge.

The dissonance engineering is the engineering science that treats on dissonance [2]. A cognitive dissonance is defined as an incoherency between individual cognitions [3]. Cindynics dissonance is a collective or an organizational dissonance related to incoherency between persons or between groups of people [4]. The occurrence of these dissonances can relate to individual 
or collective knowledge when something sounds wrong, i.e. will be, is, maybe or was wrong. A dissonant cognition is linked with contradictory information and a dissonance may produce discomfort due to the occurrence of conflicting cognition or knowledge that controls or affects behaviors, attitudes, ideas, beliefs, viewpoints, competences, etc. Dissonant knowledge of a person or of several persons can explain such conflicts. Nevertheless, dissonances can also be due to the occurrence of important or difficult decisions involving the evaluation of several possible alternatives [5], to divergent viewpoints on human behaviours [1], to the occurrence of failed competitive or cooperative activities [6], [7], to organisational changes that produce incompatible information [8], [9]. Then, the updating or the refining of a given knowledge due to new feedback from field is required but this can also generate dissonances [2].

The more difficult the learning process is to face a dissonance, the less acceptable this dissonance is. Therefore, human operators aim at reducing any occurrence or the impact of a dissonance because it produces discomfort. This activity leads to maintain a stable state of knowledge without producing any effort to change it [3]. Despite this reduction, a breakdown of this stability is sometimes useful in order to facilitate the learning process and refine, verify or confirm knowledge [10]. Such knowledge reinforcement improves the learning abilities. Finally, dissonance can also be seen as a feedback of a decision: dissonance occurs after a decision and this requires a modification of knowledge [9].

Therefore, a discomfort can be a dissonance or can be due to the production of a dissonance, and the detection or the treatment of a dissonance can also produce discomfort. Discomfort can also occur if this dissonance is over the control of the human operators or because the treatment of a detected dissonance increases the human workload or the human error for instance [11], [12]. Such an activity involves a minimum learning process in order to improve the human knowledge and to control such a discomfort. There are then positive and negative feedbacks from the dissonance management. Negative feedbacks relate to discomfort and positive ones to the learning aspect for instance.

Different structures to share tasks between human and machine such as those developed on [13], [14] can be applied for dissonance management and a learning process is usually required to facilitate the control of the knowledge content. Several models exist for self-learning, autolearning, co-learning or cooperative learning [6]. Main of them is based on the reinforcement principle by taking into account previous knowledge and integrating new or future knowledge, in order to create, modify or delete data from the knowledge base and to make it more coherent [15], [16], [17], [18].

This paper proposes an original knowledge analysis based tool to support the control of dissonances into human knowledge. It focuses on two kinds of dissonances: erroneous affordances and contradictory knowledge. This knowledge analysis is based on the knowledge modelling presented on [19]. The concept of affordances was firstly presented by Gibson [20]. Affordances can be defined as invariant relationships between direct perception and possible opportunities for action. The concept is used for different research applications related to human-machine system knowledge management [21]. For instance, the perception of a chair relates directly to the action of sitting. Regarding other human experiences, a chair can also be related to the actions of climbing when a chair is used as a ladder, or for moving or transporting when the action concerns disabled persons. The paper focuses on particular affordances: erroneous affordances when links between occurred or desired events and actions can be erroneous. The last section of this paper gives a practical example of application related to the use of an Automated Speed Control (ASC). 


\section{The knowledge analysis based tool to control dissonances}

The proposed tool analyses the content of a given knowledge base. Two modules are integrated: the module that detects possible erroneous affordances and the module that detects possible contradictory knowledge, Figure 1. The knowledge modelling and reinforcement to create, modify or delete some knowledge are allocated to the users. An interface gives the results of these detections that are validated by the users to reinforce the knowledge modelling and then the knowledge content. The knowledge analysis is based on the knowledge modelling presented on [19]. Therefore, the set of knowledge $\mathrm{K}$ contains a list of rules $R(i)$ with a condition of activation noted $\operatorname{Condit}(R(i))$ and a conclusion noted $\operatorname{Conclu}(R(i))$ :

$$
R(i) \in K \rightarrow R(i)=(\operatorname{Condit}(R(i)) \rightarrow \operatorname{Conclu}(R(i)))
$$

If $\operatorname{Condit}(R(i))$ is to be achieved or realized then $\operatorname{Conclu}(R(i))$ has to be realized. For instance, Condit $(R(i))$ can relate to a goal to be achieved or realized and $\operatorname{Conclu}(R(i))$ can correspond to the actions to be done on the process by using specific supports of the system or its environment to achieve this goal. These actions can be realized by the users of the system or by an automated module implemented into this system. Some rules are then the procedures to apply in order to achieve a goal and other rules concern the different steps required to achieve a goal. The affordance detection and the contradictory knowledge detection use the function

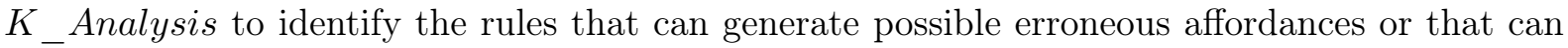
present incoherency.

The $K_{-}$Analysis function is defined as follows:

$$
\begin{aligned}
K_{-} \text {Analysis }: & K \rightarrow K \\
& R \rightarrow R^{+}=K_{-} \text {Analysis }(R), \\
& \forall R(i) \in R, \text { Condit }(R(i)) \cap B E \neq\{\emptyset\}, R^{+} \leftarrow \cup R(i)
\end{aligned}
$$

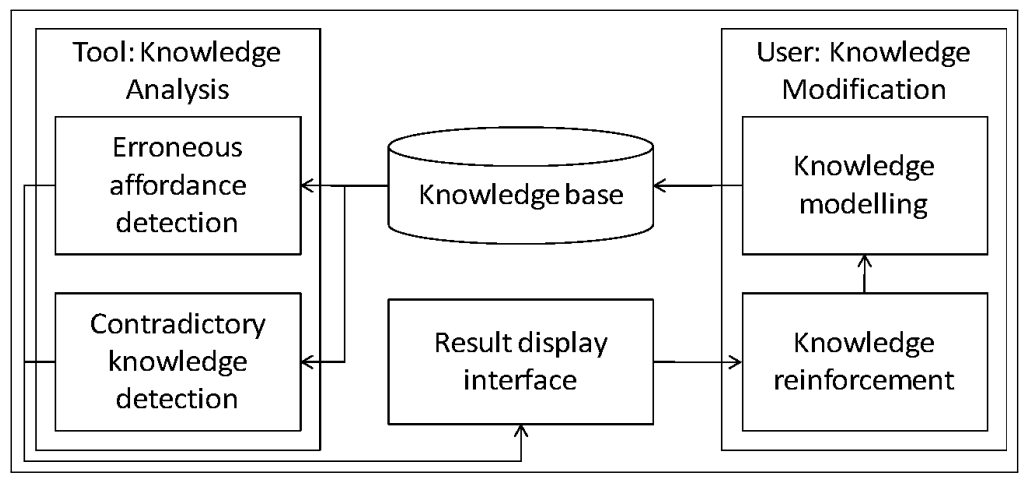

Figure 1: The automated tool modules.

The set $K$ is the set of all the possible rules. For a given rule base noted $R$ of $K$ containing a limited number of rules, the $K_{-}$Analysis gives a reduced rule base noted $R^{+}$of $K . R^{+}$contains the rules related to the inputs noted $B E$. $B E$ contains the events that occur or the goals to be achieved. When the condition $C o n d i t(R(i))$ of a rule occurs on $B E$ entirely or partially, then this rule is integrated into $R^{+}$.

The $K_{-}$Affordance function aims at identifying possible new rules combining the condition 
and the conclusion of existing rules. It is defined as follows:

$$
\begin{aligned}
K_{-} \text {Affordance }: & K \rightarrow K \\
& R \rightarrow R^{a}=K \_ \text {Affordance }(R), \\
& \forall R(i) \in R, \forall R(j) \in R, i \neq j, \operatorname{Condit}(R(i)) \subset \operatorname{Condit}(R(j)), \\
& R^{a}=\cup((\operatorname{Condit}(R(i)), \operatorname{Conclu}(R(j))),(\operatorname{Condit}(R(j)), \operatorname{Conclu}(R(i))))
\end{aligned}
$$

The result of this function is a new rule base noted $R^{a}$ of $K$ that combines conditions and conclusions of some rules of $R$. This function proposes new rules based on the affordance application concept in order to list possible new rules taking into account possible relationships between the condition of a given rule with the conclusion of another one. If a condition of a rule is included into to the condition of another one, then both rules can be used to create new rules. This process is limited to the rules identified by the $K_{-}$Analysis function. Then:

$$
R^{a}=K_{-} \text {Affordance }\left(K_{-} \text {Analysis }(R)\right)
$$

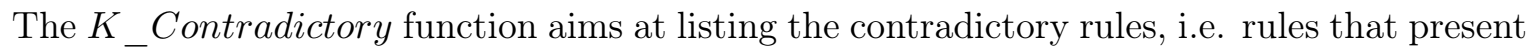
opposite behaviours. It is defined as follows:

$$
\begin{aligned}
\text { K_Contradictory }: & K \rightarrow K \\
& R \rightarrow R^{c}=K \_ \text {Contradictory }(R), \\
& \forall R(i) \in R, \forall R(j) \in R, i \neq j, \\
& (\operatorname{Conclu}(R(i)) \subset \neg \operatorname{Conclu}(R(j))) \text { or } \\
& \quad(\neg(\operatorname{Conclu}(R(i))) \subset \operatorname{Conclu}(R(j))), \\
& R^{c}=\cup(R(i),(R(j))
\end{aligned}
$$

When opposite conclusions appear on $R$, i.e. when both $\operatorname{Conclu}(R(i))$ and $\neg(\operatorname{Conclu}(R(i)))$ exist, then a possible incoherency occurs. The result of this function is a new rule base noted $R^{c}$ that contains possible conflicts between rules of $R$. This process is limited to the rules identified by the $K_{-}$Analysis function. Then:

$$
R^{c}=K_{-} \text {Contradictory }\left(K_{\text {_Analysis }}(R)\right)
$$

This formalism was applied to car driving domain by integrating into the initial rule base the rules related to the use of a Cruise/Speed Control (ASC) system. If the ASC system is activated and if an initial setpoint value is given by the car driver, the ASC has in charge the regulation of the car speed by maintaining this setpoint speed. The "+" and the "-" buttons are used for giving the initial setpoint speed or to modify this setpoint, Figure 2. The "+" button aims at increasing the setpoint value whereas the "-" button at decreasing it.

Several dissonances can generate a possible evolution of the car driver knowledge. The next two sections presents some examples of the detection of possible erroneous affordances and contradictory rules linked to the use of such a system. The wording of the rule condition and conclusion are voluntarily simple in order to illustrate the feasibility of such a knowledge analysis to detect dissonances. Perspectives will consist in using more complex rules with a numerical model integrating for instance belief on rules, belief on condition occurrence or belief on conclusion occurrence. 


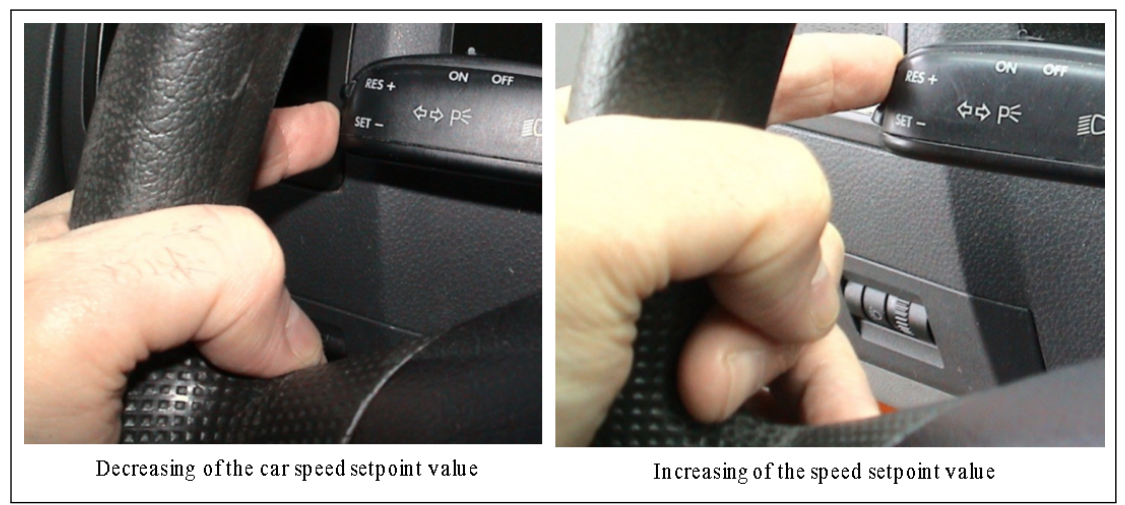

Figure 2: The "+" and "-" buttons of an ASC system of a car.

\section{Example of possible erroneous affordance detection} rules:

Suppose that a knowledge modeling process produced a knowledge base containing these

- $R(1):$ (the use of the ASC system $\rightarrow$ turn the activation button on "on")

- $R(2)$ : (the deactivation of the ASC system $\rightarrow$ brake with the braking pedal)

- $R(3)$ : (the increasing of the car speed setpoint $\rightarrow$ push the " + " button)

- $R(4)$ : (the decreasing of the car speed setpoint $\rightarrow$ push the "-" button)

- $R(5)$ : (the increasing of the car speed $\rightarrow$ push the gas pedal)

- $R(6)$ : (the decreasing of the car speed $\rightarrow$ release the gas pedal)

For instance, whatever the context of the ASC system use, if $B E$ contains initially increasing of the car speed, $R^{a}$ will list several dissonances to be tested or validated by the car driver. It will contain two possible new rules:

- (the increasing of the car speed setpoint $\rightarrow$ push the gas pedal)

- (the increasing of the car speed $\rightarrow$ push the " + " button)

If $B E$ contains initially "decreasing of the car speed", $R^{a}$ will then contain other new possible dissonances:

- (the decreasing of the car speed setpoint $\rightarrow$ release the gas pedal)

- (the decreasing of the car speed $\rightarrow$ push the "-" button)

10 subjects who usually used an ASC system were invited to evaluate the proposed erroneous affordances, Table 1. The outputs of the automated systems were presented to these subjects who have to make comments about them. They have to give their own point of view about the dissonances.

All of them considered that the rules related to the management of the car speed setpoint value are erroneous affordances. However, 8 of them do not consider the other rules as problems and decided to integrate the proposed rules into the knowledge base content. Therefore, these subjects consider that they can manage the car speed without taking into account the management of 
Table 1: Subjective evaluation of the proposed erroneous affordances.

\begin{tabular}{|l|l|l|}
\hline Proposed erroneous affordance & $\begin{array}{l}\text { Real erroneous affor- } \\
\text { dance? }\end{array}$ & Consequences \\
\hline $\begin{array}{l}\text { (the increasing of the car speed setpoint } \\
\rightarrow \text { push the gas pedal) }\end{array}$ & $\begin{array}{l}\text { Yes (10 upon 10 sub- } \\
\text { jects) }\end{array}$ & $\begin{array}{l}\text { No modification of the } \\
\text { knowledge base }\end{array}$ \\
\hline $\begin{array}{l}\text { (the increasing of the car speed } \rightarrow \text { push } \\
\text { the "+" button) }\end{array}$ & No (8 upon 10 subjects) & $\begin{array}{l}\text { Creation of this rule into } \\
\text { the knowledge base }\end{array}$ \\
\hline $\begin{array}{l}\text { (the decreasing of the car speed setpoint } \\
\rightarrow \text { release the gas pedal) }\end{array}$ & $\begin{array}{l}\text { Yes (10 upon 10 sub- } \\
\text { jects) }\end{array}$ & $\begin{array}{l}\text { No modification of the } \\
\text { knowledge base }\end{array}$ \\
\hline $\begin{array}{l}\text { (the decreasing of the car speed } \rightarrow \text { push } \\
\text { the "-" button) }\end{array}$ & No (8 upon 10 subjects) & $\begin{array}{l}\text { Creation of this rule into } \\
\text { the knowledge base }\end{array}$ \\
\hline
\end{tabular}

the pedals anymore. This reduces their workload. The 2 subjects who did not accept these new rules consider these rules as dangerous because this can lead to adapt a possible body position that can generate problems in case of emergency stop for instance. Figure 3 gives an example of such a body position into the car when managing the car speed only with the "+" and "-" buttons of the ASC system: the legs are crossed because the car speed is managed by a finger that activates the "+" or ""-" buttons, and the position of the legs can therefore become an obstacle or a discomfort in case of emergency stop that may require a quick press on the brake pedal!

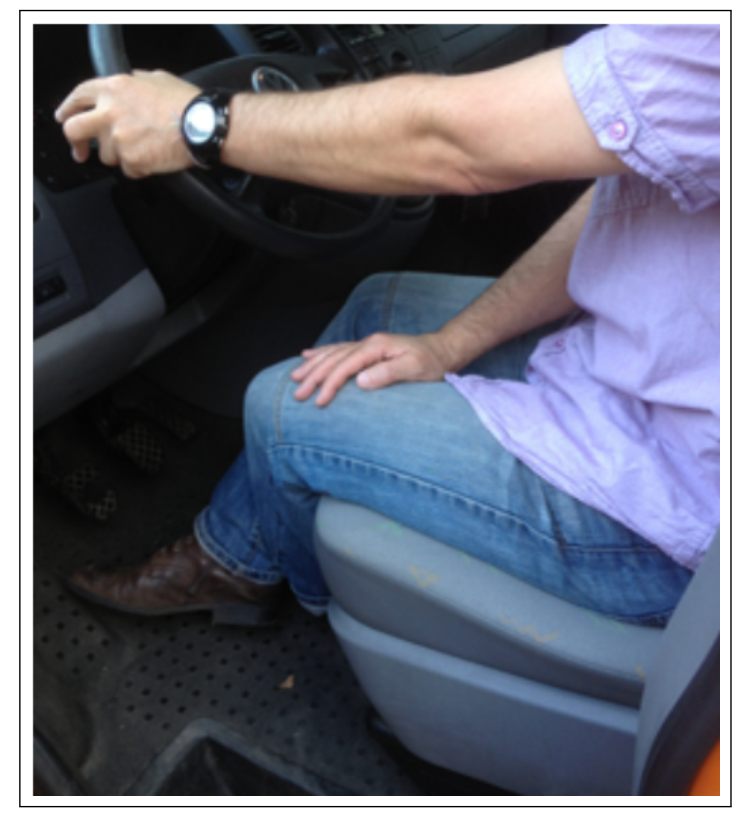

Figure 3: Example of a possible dangerous body position when applying the proposed rules for regulating the car speed.

\section{Example of possible contradictory knowledge detection}

Suppose that, for another use context, the knowledge modelling process produced another knowledge base combining rules related to the aquaplaning control and some rules related to the ACS control: 
- $R(1)$ : (the use of the ASC system $\rightarrow$ turn the activation button "on")

- $R(2)$ : (the deactivation of the ASC system $\rightarrow$ brake)

- $R(3)$ : (the reduction of the current car speed that becomes under the setpoint managed by the ASC $\rightarrow$ accelerate automatically to reach the setpoint value)

- $R(4)$ ): (the increasing of the current car speed that becomes over the setpoint managed by the ASC $\rightarrow$ decelerate automatically to reach the setpoint value)

- $R(5)$ : (the control of an aquaplaning $\rightarrow \neg($ brake))

- $R(6)$ : (the control of an aquaplaning $\rightarrow \neg$ (accelerate))

Knowing that the ASC is activated, the car speed setpoint is high and the current car speed is equal to the required setpoint, the occurrence of an aquaplaning may reduce the current car speed due to the natural braking and friction related to the water level on the road. Suppose that in this case, the initial $B E$ content is (control of aquaplaning, deactivation of the ACS, reduction of the current car speed). The contradictory knowledge module identifies some couples of possible dissonant rules. $R^{c}$ will contains this list of couples of rules:

- $(($ the deactivation of the ASC system $\rightarrow$ brake), (the control of an aquaplaning $\rightarrow \neg($ brake $)))$

- ((the control of an aquaplaning $\rightarrow \neg$ (accelerate)), (the reduction of the current car speed that becomes under the setpoint managed by the ASC $\rightarrow$ accelerate automatically to reach the setpoint value))

10 subjects were invited to assess these contradictory knowledge proposals. They use an ACS system and are aware about the behaviour to follow and about the car behaviour when an aquaplaning occurs. All of them are agree with the contradictory rules proposed by the automated system, Table 2 .

Table 2: Subjective evaluation of the proposed contradictory knowledge.

\begin{tabular}{|l|l|l|}
\hline Proposed contradictory knowledge & $\begin{array}{l}\text { Real contradictory } \\
\text { knowledge? }\end{array}$ & Consequences \\
\hline $\begin{array}{l}((\text { the deactivation of the ASC system } \rightarrow \\
\text { brake), (the control of an aquaplaning } \rightarrow \\
\neg(\text { brake }))\end{array}$ & $\begin{array}{l}\text { Yes }(10 \text { upon 10 sub- } \\
\text { jects) }\end{array}$ & $\begin{array}{l}\text { Modification of the cur- } \\
\text { rent rules or creation of } \\
\text { new rules }\end{array}$ \\
\hline $\begin{array}{l}((\text { the control of an aquaplaning } \rightarrow \\
\neg(\text { accelerate })),(\text { the reduction of the cur- } \\
\text { rent car speed that becomes under the set- } \\
\text { point managed by the ASC } \rightarrow \text { accelerate } \\
\text { automatically to reach the setpoint value })\end{array}$ & jects $)$ & $\begin{array}{l}\text { Modification of the cur- } \\
\text { rent rules or creation of } \\
\text { new rules }\end{array}$ \\
\hline
\end{tabular}

The contradictory actions (brake, $\neg$ (brake)) and (accelerate, $\neg$ (accelerate)) are then solved by reinforcing the knowledge in different possible ways such as the modification of some current rules or the creation of new rules. They recognize that in emergency situations, it is more natural to use the braking pedal for stopping the car or deactivating a system instead of using the clutch pedal or the "off" button of the ASC system as it is noted on the ASC user manual. Some user manual recommends using the system in particular conditions. For instance, it indicates not to use the system when it is raining. However, water can sometimes occur under bridges for instance even if it is not raining and this can generate aquaplaning. 


\section{Conclusion}

This paper is an original contribution on risk analysis based on dissonance engineering. It proposes a knowledge analysis based tool composed by two main modules: an erroneous affordances detection module, and a contradictory knowledge detection module.

The knowledge analysis consists in identifying possible dissonances into a knowledge base composed by rules. Rules contain conditions of activation and conclusions when they can be activated. The conditions relate to occurred or desired events and the conclusions to the associated actions to be achieved. The erroneous affordances module treats particular dissonances that the users may create. They are new rules for which desired or occurred events may be related to wrong actions. These possible erroneous relations between events and actions are obtained by using the existing rules and initial events. The contradictory knowledge module manages the rules for which the activation presents opposite actions regarding initial inputs.

A practical example is then proposed to study the feasibility of use of such a knowledge analysis based tool. It relates to the use of an Automated Speed Control systems dedicated to car driving. Rules associated to its use and other rules applied for controlling events such as an aquaplaning were proposed. Erroneous affordances and contradictory knowledge are then given by the proposed automated modules. 10 subjects were invited to make comments about these outputs from the proposed tool.

Among the erroneous affordances proposed by the system, there is a rule that was not considered as wrong and that was accepted by 8 subjects upon 10 . It concerns the use of the "+" and "-" buttons of the ASC in order to increase or decrease respectively the current car speed on demand instead of using these buttons to control a stable car speed setpoint. The acceleration and deceleration on road or motorway can then be done with such new procedures without involving the legs anymore. The 2 last subjects that agree with the system consider this new rule as dangerous because problems may occur if an emergency stop is required for instance. Problems can be related to the position of the legs or to the body into the car due to the new function allocated to the "+" and "-" buttons for controlling the car speed.

Regarding the contradictory knowledge, all the subjects were agree with the proposals they consider as very dangerous. Indeed, they considered that, in emergency case, people may activate the braking pedal instead of the "off" button of the ASC system or the clutch pedal to deactivate the ASC system. Then, the rules associated to the control of aquaplaning that required no action on the braking pedal and no action on the speed control are contradictory with rules related to the use or behavioural model of the ASC system.

This simple example has shown the interest of such a new approach to analysis risks involving rule based knowledge. Future work research will then focus on more complex applications implementing numerical models of knowledge and will integrate criteria such as uncertainties, beliefs or preferences on knowledge [22], [23]. Future applications will connect automated reinforced learning systems to assist the knowledge reinforcement process. Finally, this paper is a call to future designers of car driving systems such as ASC systems to use a dissonance engineering based risk analysis for designing system functions and user manuals in order to control possible dangerous dissonances and recover side effects of automation.

\section{Acknowledgments}

The present research work has been supported by the International Research Group on Human-Machine Systems in Transportation and Industry: the author gratefully acknowledges the support of this institution. The author thanks also P. Richard for his support to format this paper. 


\section{Bibliography}

[1] F. Vanderhaegen (2010), Human-error-based design of barriers and analysis of their uses Cognition Technology \& Work, 12: 133-142

[2] F. Vanderhaegen (2013), A Dissonance Management Model for Risk Analysis Proceedings of the 12th IFAC/IFIP/IFORS/IEA Symposium on Analysis, Design, and Evaluation of Human-Machine Systemsl, Las Vegas, USA, August, 11-15.

[3] L. Festinger (1957), A theory of cognitive dissonance. Stanford, CA: Stanford University Press.

[4] G.-Y. Kervern (1995), Eléments fondamentaux des cindyniques (Fondamental elements of cindynics) Economica Editions, Paris.

[5] T.-Y. Chen (2011), Optimistic and pessimistic decision making with dissonance reduction using interval-valued fuzzy sets Information Sciences, 181(3): 479-502.

[6] F. Vanderhaegen (2012), Cooperation and learning to increase the autonomy of ADAS Cognition, Technology \&5 Work, 14: 61-69.

[7] F. Vanderhaegen, S. ChalmĂ ̌̌, F. Anceaux, P. Millot (2006), Principles of cooperation and competition - Application to car driver behavior analysis Cognition, Technology 83 Work, 8(3): 183-192.

[8] O. Brunel, C. Gallen (2011), Just like cognitive dissonance Proceedings of the 27th International Congress of French Association of Marketing, 8-20 May 2011, Brussels.

[9] E. E. Telci, C. Maden, D. Kantur (2011), The theory of cognitive dissonance: A marketing and management perspective Procedia Social and Behavioral Sciences, 24: 378-386.

[10] E. AĂŻmeur (1998), Application and assessment of cognitive dissonance Theory in the learning process Journal of Universal Computer Science, 4(3): 216-247.

[11] F. Vanderhaegen (1999), Cooperative system organisation and task allocation: illustration of task allocation in air traffic control Le Travail Humain, 63(3): 197-222.

[12] F. Vanderhaegen (1999), Multilevel allocation modes - Allocator control policies to share tasks between human and computer System Analysis Modelling Simulation, 35: 191-213.

[13] F. Vanderhaegen (1997), Multilevel organization design: the case of the air traffic control Control Engineering Practice, 5(3): 391-399.

[14] S. Zieba, P. Polet, F. Vanderhaegen (2011), Using adjustable autonomy and human machine cooperation to make a human machine system resilient Application to a ground robotic Information Sciences, 181(3): 379-397.

[15] F. Vanderhaegen, S. Zieba, S. Enjalbert, P. Polet (2011), A Benefit/Cost/Deficit (BCD) model for learning from human errors Reliability Engineering 83 System Safety, 96(7): 75776.

[16] P. Polet, F. Vanderhaegen, S. Zieba (2012), Iterative learning control based tools to learn from human error Engineering Applications of Artificial Intelligence, 25(7): 1515-1522. 
[17] M. Ercan, T. Acarman (2013), Processing capacity and response time enhancement by using iterative learning approach with an application to insurance policy server operation International Journal of Computers Communications \& Control, 8(4): 514-524.

[18] C.K. Ang, S.H. Tang, S. Mashohor, M.K.A.M. Arrn (2014), Solving continuous trajectory and forward kinematics simultaneously based on ANN International Journal of Computers Communications \& Control, 9(3):253-260.

[19] F. Vanderhaegen, P. Caulier (2011), A multi-viewpoint system to support abductive reasoning Information Sciences, 181(24): 5349-5363.

[20] JJ Gibson (1986), The ecological approach to visual perception Lawrence Erlbaum Associates, Hillsdale (Originally published in 1979).

[21] S. Zieba, T. Inagaki, F. Vanderhaegen (2010), Resilience engineering by the management of affordances Application to intelligent transport system. Proceedings of the 11th IFAC/IFIP/IFORS/IEA Symposium on Analysis, Design, and Evaluation of Human-Machine Systems, Valenciennes, France, August 31 September 3, 2010.

[22] F. Aguirre, M. Sallak, F. Vanderhaegen, D. Berdjag (2013), An evidential network approach to support uncertain multiviewpoint abductive reasoning Information Sciences, 253:110-125.

[23] F. Vanderhaegen, P. Polet, S. Zieba (2009), A reinforced iterative formalism to learn from human errors and uncertainty Engineering Applications of Artificial Intelligence, 22(4-5): 654-659. 